\title{
NOTE OF CONCERN
}

It has been brought to our attention that Figure 3 of "Constitutive Nuclear Factor- $\kappa B$ Activity Is Crucial for Human Retinoblastoma Cell Viability," by Vassiliki Poulaki, Constantine S. Mitsiades, Antonia M. Joussen, Alexandra Lappas, Bernd Kirchhof, and Nicholas Mitsiades (Volume 161, pages 2229-2240 of the December 2002 issue of The American Journal of Pathology) contains errors, specifically that panels $\mathrm{C}$ and D share significant overlap with panels $\mathrm{E}$ and $\mathrm{F}$, respectively, suggesting that they did not originate from different specimens. The matter was reviewed by the journal's Editors.

This matter was simultaneously brought to the attention of the Editors by corresponding author Dr. Poulaki and by a concerned reader. Dr. Poulaki states, "We believe that the most likely explanation for this error is an inadvertent misidentification of the original files at the stage of image capture with Openlab software (PerkinElmer, Waltham, MA; circa 2001), which subsequently escaped our attention due to the quantitative similarity of panels $B-F$. We sincerely regret this inadvertent error."

Dr. Poulaki adds further that "the TUNEL (terminal deoxynucleotidyl transferase-mediated dUTP nick-end labelling) assay in Figure 3 was used as an additional methodology to visually illustrate the finding of the MTT assay shown in Figure 4, namely that none of the caspase inhibitors used (ZVAD-FMK, DEVD-FMK, LEHD-FMK, IETD-FMK) can rescue the Y79 cells from SN50-induced cell death. This finding is also supported by the lack of caspase-9 cleavage or classical $85-\mathrm{kDa}$ poly (ADP-ribose) polymerase cleavage fragment (Figure 5). Thus, the TUNEL assay was only one of several methodologies used in the paper with concordant results. We are therefore confident that the conclusions of our manuscript remain unaffected by this inadvertent error and are solid and reproducible." 